\title{
Internações e Mortes por Causas Externas entre os Menores de um Ano, Recife, Pernambuco
}

\author{
Hospitalizations and Deaths by External Causes among children \\ under 1 year old, Recife, Pernambuco
}

\author{
Henry Johnson Passos de Oliveira ${ }^{1}$ \\ Victor Manoel Pereira da Silva ${ }^{2}$ \\ Raphael Alves da Silva ${ }^{3}$ \\ Felicialle Pereira da Silva ${ }^{4}$ \\ Cristine Vieira do Bonfim ${ }^{5}$
}

\section{RESUMO}

Objetivo: Descrever as características clínicas e epidemiológicas dos óbitos infantis por causas externas de residentes no Recife, Pernambuco, no período de 2010 a 2016. Metodologia: Estudo transversal, descritivo, com abordagem quantitativa. A população do estudo foi constituída por 83 óbitos infantis por causa externa de residentes no Recife. Utilizou-se o programa $R$ versão 3.6.3 para análise descritiva dos dados. Além disso, utilizou-se o teste de $\mathrm{x}^{2}$ para testar se os dados categóricos associados a uma variável se comportam de modo homogêneo. Resultados: No período de estudo foram registrados 83 óbitos infantis por causa externa em residentes na Cidade do Recife - PE. Os óbitos apresentaram maiores proporções em indivíduos do sexo masculino $(n=44 ; 53,0 \%)$, da raça/cor preta ( $n$ $=54 ; 65,1 \%)$ no período pós-neonatal $(n=65 ; 78,3 \%)$. A maior parte dos óbitos foi em decorrência de acidentes envolvendo a respiração, cuja principal causa básica foi inalação do conteúdo gástrico ( $n=47 ; 56,6 \%)$. Conclusão: $A$ análise descritiva das características dos óbitos infantis por causa externa possibilita que ações de políticas públicas e efetivas sejam planejadas segundo a multiplicidade de fatores que determinam o risco de morte por causa externa entre os menores de um ano.

\section{DESCRITORES}

Causas Externas. Mortalidade Infantil. Lesões e Ferimentos.

\begin{abstract}
Objective: To describe the clinical and epidemiological characteristics of infant deaths due to external causes of residents in Recife, Pernambuco, from 2010 to 2016. Methodology: Cross-sectional, descriptive study with a quantitative approach. The study population consisted of 83 infant deaths due to external causes of residents in Recife. The $\mathrm{R}$ version 3.6.3 program was used for descriptive analysis of the data. In addition, the $\mathrm{x}^{2}$ test was used to test whether the categorical data associated with a variable behaved homogeneously. Results: During the study period, 83 infant deaths due to external causes were recorded in residents of the city of Recife - PE. Deaths showed higher proportions in males $(n=44 ; 53.0 \%)$, of black race/color $(n=54 ; 65.1 \%)$, in the post-neonatal period $(n=65$; $78.3 \%$ ). Most deaths were due to accidents involving breathing, the main underlying cause being inhalation of gastric contents $(n=47 ; 56.6 \%)$. Conclusion: The descriptive analysis of the characteristics of infant deaths due to external causes allows public and effective policy actions to be planned according to the multiplicity of factors that determine the risk of death from external causes among children under one year old.
\end{abstract}

\section{DESCRIPTORS}

External Causes. Child Mortality. Injuries and Wounds.

${ }^{1}$ Enfermeiro. Residente em Saúde Coletiva pelo Instituto Aggeu Magalhães - FIOCRUZ-PE, Recife, Pernambuco, Brasil.

${ }^{2}$ Graduando em Enfermagem pela Faculdade dos Guararapes, Recife, Pernambuco, Brasil.

${ }^{3}$ Enfermeiro. Centro Universitário Maurício de Nassau - UNINASSAU, Recife, Pernambuco, Brasil.

${ }^{4}$ Enfermeira. Doutora em Neuropsiquiatria e Ciências do Comportamento pela Universidade Federal de Pernambuco e Professora Adjunta da Faculdade de Enfermagem Nossa Senhora das Graças da Universidade de Pernambuco, Recife, Pernambuco, Brasil.

${ }^{5}$ Sanitarista. Doutora em Saúde Pública pelo Instituto de Pesquisa Aggeu Magalhães - FIOCRUZ. Pesquisadora da Fundação Joaquim Nabuco, Recife, Pernambuco, Brasil. 
A causas externas figuram entre os principais responsáveis por internamentos e mortes entre crianças e adolescentes no mundo. Entre as causas de mortalidade, o óbito infantil por causas externas, derivada de acidentes, violências ou tratamento médico ineficiente, caracteriza-se como evitável, visto que, poderia ser reduzido por meio de ações de serviços de saúde acessíveis e efetivos ${ }^{1}$.

Os acidentes constituem um problema de saúde pública mundial ${ }^{2}$. Um estudo realizado no Japão evidenciou que, entre os nascidos vivos entre 2003 a 2010, 1.194 morreram acidentalmente antes do seu primeiro ano de vida. No Brasil, em 2016 foram registrados 1.091 óbitos por causas externas entre os menores de um ano. Dentre estes; 636 mortes foram ocasionadas por sufocação ou engasgamento ${ }^{3}$.

A morte infantil, em muitos casos, não se deve a uma única causa, mas a uma complexa soma de fatores de risco. A qualidade, a disponibilidade e o acesso aos cuidados em saúde são aspectos importantes que podem influenciar não apenas a probabilidade de sobreviver a uma lesão, mas, também, as consequências em longo prazo ${ }^{4-6}$. Programas de prevenção que levam em consideração vulnerabilidades e usam uma abordagem multidisciplinar têm se mostrado os mais eficazes para reduzir a mortalidade infantil como resultado de uma lesão ${ }^{7}$.

O Sistema de Informações Sobre Nascidos Vivos (SINASC) e o Sistema de Informações sobre Mortalidade (SIM) configuram-se como fontes de dados dos serviços de saúde indispensáveis para o monitoramento epidemiológico da mortalidade infantil. A análise de bancos de dados nos permite avaliar mudanças nos perfis de mortalidade e, consequentemente, possibilita desenvolver estratégias preventivas direcionadas à redução do risco de óbito por causa externa entre menores de um ano.

Nesta perspectiva, o presente estudo tem como objetivo, descrever as características clínicas e epidemiológicas dos óbitos infantis por causas externas de residentes no Recife, Pernambuco, no período de 2010 a 2016.

\section{METODOLOGIA}

Trata-se de um estudo transversal, descritivo, com abordagem quantitativa, realizado na Cidade do Recife, Pernambuco, Brasil. A população do estudo foi constituída por 83 óbitos infantis por causa externa de residentes no Recife entre os anos de 2010 e 2016. A fonte de dados foi constituída pela consulta ao Sistema de Informação sobre Mortalidade e Sistema de Informação sobre Nascidos Vivos.

Foram investigadas 16 variáveis. Agrupou-se a raça/cor em (brancos ou pretos - (negros e pardos); idade da mãe (<20 anos; $\geq 20$ anos) e a escolaridade (< oito anos de estudo; $\geq$ oito anos de estudos). A variável idade foi categorizada em neonatal precoce $(0$ a 6 dias de vida), neonatal tardio (7 a 27 dias) e pós-neonatal (28 a menos de um ano). Para o cálculo do coeficiente de mortalidade infantil por causa externa, utilizou-se da razão entre o número $B$ de óbitos de menores de um ano de idade e o número de nascidos vivos em um determinado ano e local x 1000 .

Para avaliação da causa básica de morte utilizou-se a $10^{a}$ revisão da Classificação Estatística Internacional de Doenças e Problemas Relacionados à Saúde (CID - 10). Para definição de evitabilidade adotou-se a lista de causas de mortes evitáveis por inter- 
venções do Sistema Único de Saúde (SUS) ${ }^{8}$.

Utilizou-se o programa $R$ versão 3.6.3 para análise descritiva dos dados, por meio da distribuição das frequências absolutas e relativas. Além disso, foi utilizado o teste de $\mathrm{x}^{2}$ para testar se os dados categóricos associados a uma variável se comportam de modo homogêneo. A homogeneidade foi descartada quando $p$-valor $<0,05$.

A execução deste estudo obedeceu às normas estabelecidas pela Resolução $n^{\circ}$. 466/12, do Conselho Nacional de Saúde que trata sobre os aspectos éticos com pesquisa envolvendo seres humanos. A anuência foi solicitada à Diretoria Executiva de Gestão do Trabalho e Educação na Saúde por meio do Sistema de Cadastro de Projeto de Pesquisa e/ou Extensão da Secretaria do Recife. O projeto de pesquisa foi submetido para avaliação do Comitê de Ética em Pesquisas em Seres Humanos da Fundação Joaquim Nabuco e aprovado sob Parecer $n^{\circ}$. 3.355.528 (CAAE: 13326519.3.0000.5619).

\section{RESULTADOS}

\section{Gráfico 1. Coeficiente de mortalidade infantil por causa externa}

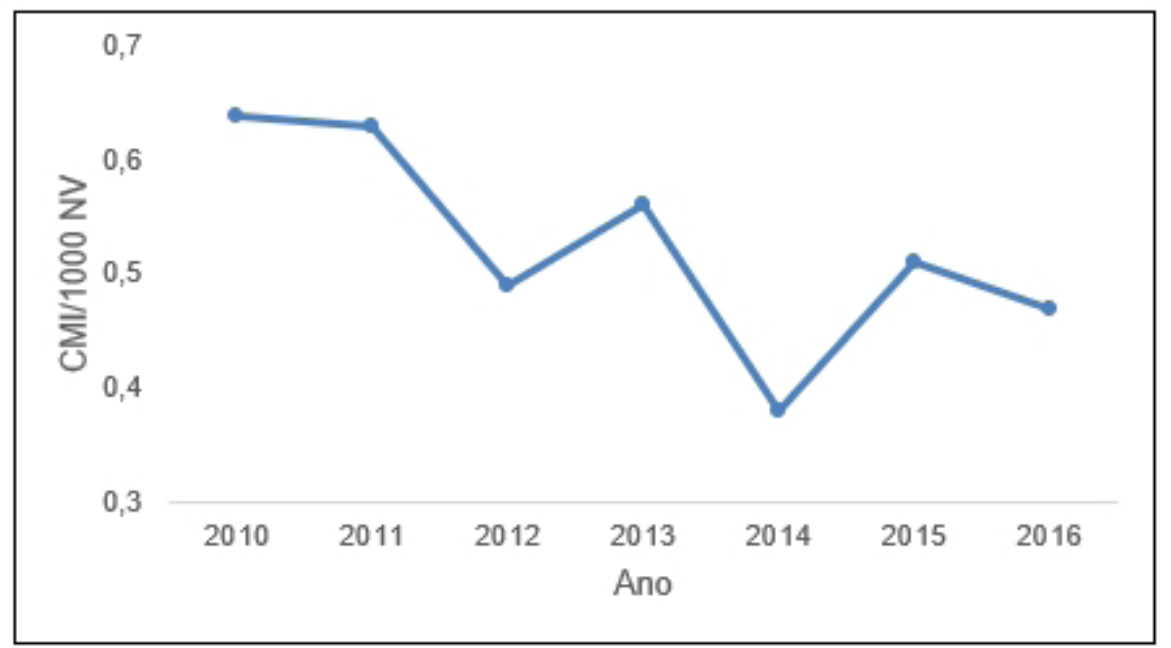


Tabela 1. Caracterização das variáveis associadas aos óbitos infantis por causa externa

\begin{tabular}{|c|c|c|c|}
\hline Variáveis & (n) & (\%) & (p-valor) \\
\hline Sexo & 44 & 53 & \\
\hline Masculino & 44 & 53 & \\
\hline Feminino & 39 & 47 & 0,5831 \\
\hline Raça/cor ${ }^{(a)}$ & & & \\
\hline Branco & 23 & 27,7 & \\
\hline Preto & 54 & 65,1 & $<0,001$ \\
\hline Componente etário & & & \\
\hline Neonatal precoce & 06 & 7,2 & \\
\hline Neonatal tardio & 12 & 14,5 & \\
\hline Pós neonatal & 65 & 78,3 & $<0,001$ \\
\hline Local de ocorrência do óbito & & & \\
\hline Hospital & 57 & 68,7 & \\
\hline Outro estabelecimento de saúde & 01 & 1,2 & \\
\hline Domicilio & 17 & 20,5 & \\
\hline Via pública & 07 & 8,4 & \\
\hline Outros & 01 & 1,2 & $<0,001$ \\
\hline Circunstância do óbito(b) & & & \\
\hline Acidente & 46 & 55,4 & \\
\hline Homicidio & 03 & 3,6 & \\
\hline Outros & 03 & 3,6 & $<0,001$ \\
\hline Investigação Epidemiológica & & & \\
\hline Sim & 79 & 95,2 & \\
\hline Não & 04 & 4,8 & $<0,001$ \\
\hline A investigação alterou a causa básica & & & \\
\hline Sim & 43 & 54,4 & \\
\hline Não & 36 & 45,6 & \\
\hline Evitabilidade & & & \\
\hline Sim & 83 & 100,0 & \\
\hline Não & - & - & $<0,001$ \\
\hline Total & 83 & 100 & \\
\hline
\end{tabular}

uma gravidez única $(n=75 ; 90,4 \%)$ com parto vaginal $(n=44 ; 53,0 \%)$ conforme apresentado na Tabela 2.

A maior parte dos óbitos foi em decorrência de acidentes envolvendo a respiração, cuja principal causa básica foi inalação do conteúdo gástrico ( $n=47 ; 56,6 \%$ ), conforme apresentado na Tabela 3.

\section{DISCUSSÃO}

Os resultados do estudo mostraram que ocorreu uma redução do coeficiente de mortalidade infantil por causa externa na Cidade do Recife. Além da ampliação de políticas públicas intersetoriais e ações em serviços de saúde, parte dessa redução se deu a partir da implementação da vigilância do óbito infantil. Esta prática favoreceu a compreensão das circunstâncias em que o evento ocorreu e forneceu informações confiáveis que permitem o correto diagnóstico da situação de saúde e das características dos diferentes extratos sociais ${ }^{9}$.

Dentre as características dos óbitos, o sexo masculino foi predominante nos óbitos infantis por causa externa entre os menores de um ano. Atribui-se essa predominância às diferenças comportamentais de cada sexo e aos fatores culturais que determinam maior vigilância sobre as meninas. Além disso, sugere-se que o indivíduo do sexo masculino 
Tabela 2. Caracterização das variáveis associadas às mães das crianças menores de um ano que foram a óbito por causa externa

\begin{tabular}{|c|c|c|c|}
\hline Variáveis & (n) & $(\%)$ & (p-valor) \\
\hline \multicolumn{4}{|l|}{ Idade (a) } \\
\hline $\begin{array}{l}19 \text { ou menos } \\
20 \text { ou mais }\end{array}$ & $\begin{array}{l}16 \\
64\end{array}$ & $\begin{array}{l}19,3 \\
77,1\end{array}$ & $<0,001$ \\
\hline Escolaridade (b) & & & \multirow{3}{*}{0,7357} \\
\hline Menos de 8 anos & 39 & 47,0 & \\
\hline 8 ou mais anos & 41 & 49,4 & \\
\hline Filhos vivos (c) & & & \multirow{3}{*}{$<0,001$} \\
\hline Sim & 70 & 84,3 & \\
\hline Não & 10 & 12,0 & \\
\hline Filhos mortos (d) & & & \multirow{3}{*}{$<0,001$} \\
\hline Sim & 17 & 20,5 & \\
\hline Não & 62 & 74,7 & \\
\hline Tipo de gravidez (e) & & & \multirow{4}{*}{$<0,001$} \\
\hline Unica & 75 & 90,4 & \\
\hline Dupla & 03 & 3,6 & \\
\hline Tripla & 01 & 1,2 & \\
\hline Tipo de parto (f) & & & \multirow{3}{*}{0,2575} \\
\hline Vaginal & 44 & 53,0 & \\
\hline Cesário & 34 & 41,0 & \\
\hline Total & 83 & 100 & \\
\hline
\end{tabular}

Tabela 3. Caracterização da causa básica dos óbitos infantis por causa externa

\begin{tabular}{lcc}
\hline \multicolumn{1}{c}{ Variável } & (n) & (\%) \\
\hline Causa básica & 01 & 1,2 \\
W18 - Outras quedas no mesmo nível & 02 & 2,4 \\
W77 - Risco a respiração devido a desmoronamento, queda de terra e outras & 47 & 56,6 \\
substâncias & 02 & 2,4 \\
W78 - Inalação do conteúdo gástrico & 01 & 1,2 \\
W79 - Inalação e ingestão de alimentos causando obstrução do trato respiratório & 01 & 1,2 \\
W80 - Inalação e ingestão de outros objetos causando obstrução do trato respiratório & 01 & 1,2 \\
W84 - Riscos não especificados à respiração & 01 & 1,2 \\
W87 - Exposição a corrente elétrica não especificada & 01 & 1,2 \\
X06 - Exposição a combustão de outro tipo de roupa ou de acessórios & 01 & 1,2 \\
X09 - Exposição a tipo não especificado de fumaça, fogo ou chamas & 01 & 1,2 \\
X58 - Exposição a outros fatores não especificados & 01 & 1,2 \\
X91 - Agressão por meio de enforcamento, estrangulamento e sufocação & 01 \\
X95 - Agressão por meio de disparo de outra arma de fogo ou de arma não & 1,2 \\
especificada & 08 \\
Y06 - Negligência e abandono & 9,6 \\
Y21 - Afogamento e submersão, intenção não determinada & 14 & 16,9 \\
Y34 - Fatos ou eventos não especificados e intenção não determinada & 83 & 100 \\
\hline Total &
\end{tabular}


possua maior perímetro cefálico, o que ocasiona maior desequilíbrio corpóreo no início dos seus movimentos motores e, consequentemente, maior risco à queda ${ }^{9,10}$.

A raça/cor preta apresentou destaque nos óbitos de menores de um ano. Atribui-se a raça/cor preta como detentora de maior risco ao óbito infanti ${ }^{8-10}$. As desigualdades segundo raça/cor manifestam em diversos indicadores, parecem espelhar o cenário maior de exclusão e iniquidades ao acesso aos serviços de saúde, além da precariedade nas condições socioeconômicas, ambientais e sanitárias as quais esta população é submetida ao longo de suas vidas ${ }^{11}$. No Brasil, a escravidão marcou profundamente a posição social de sucessivas gerações. No entanto, a raça/cor em si não é um fator de risco e, sim, a inserção social adversa de um grupo racial/étnico que constitui característica de vulnerabilidade ${ }^{12}$.

Os óbitos ocorreram, em sua maioria, no ambiente hospitalar, o que reflete acesso da população estudada aos serviços de saúde. Além disso, semelhante aos resultados descritos em uma pesquisa realizada nos Estados Unidos, os óbitos se agruparam no período pós-neonatal ${ }^{13}$. Atribui-se ao fato de que no período pós-neonatal, a criança apresenta maior desenvolvimento motor, sujeitando-os às lesões decorrentes de acidentes ${ }^{14}$. Também é neste período que se inicia a introdução alimentar, que pode predispor aos maiores riscos de acidentes envolvendo a inalação do conteúdo gástrico; ou de corpo estranho (broncoaspiração) $^{15}$.

A maioria dos óbitos ocorreu de forma acidental. A inalação do conteúdo gástrico foi a principal causa básica. Autores abordaram os desfechos associados à sufocação como a causa externa mais comum de óbito infantil $^{16,17}$. A introdução alimentar e a interrupção da amamentação exclusiva contribuíram para uma maior ocorrência de óbitos infantis decorrentes de broncoaspiração. Verifica-se que, pelo fato da criança não possuir a dentição posterior completa, dificulta o ato de mastigar e engolir, aumentando o risco de broncoaspirar ${ }^{18}$.

Em relação às características maternas, a maior concentração de óbitos se deu em crianças de mães com idade superior a 20 anos. Nos últimos anos, ocorreu uma maior inserção da mulher no ambiente escolar e profissional. Com maior renda e maior instrução educacional. Dessa forma, observa-se redução da gestação entre adolescentes ${ }^{19-21}$.

Entre os achados dessa pesquisa, observou-se maior periodicidade de óbitos entre os bebês de mães com oito ou mais anos de estudo. Nas últimas décadas ocorreu uma expansão e melhoria no processo educacional e, consequentemente, maior escolarização das sociedades ${ }^{22,23}$. Afirma-se que o grau de escolaridade materna é diretamente proporcional ao grau de conhecimento sobre o cuidado que poderia vir a reduzir o risco de acidentes. Além disso, o baixo nível de conhecimento pode inibir a procura por atendimento médico ${ }^{24-26}$. A maior parte das mulheres possuía outros filhos e não tinha histórico de óbito infantil. Uma pesquisa realizada em Passo Fundo RS relatou que a quantidade de filhos vivos é um determinante de risco para óbito infantil em geral. Os lares com maior quantidade de crianças diminuem a vigilância dos genitores sobre as mesmas, assim, podem potencializar a ocorrência de lesões por causas externas ${ }^{27}$.

Autores evidenciam a importância da educação em saúde no pré-natal como forma de prevenção aos acidentes no primeiro ano de vida ${ }^{15,16,18}$. Nesse momento, as mães aprendem sobre a melhor posição para be- 
bês dormirem a fim de evitar a morte súbita por interrupção da ventilação. Além disso, a pega correta na amamentação, bem como a importância da exclusividade até o sexto mês de vida deve ser evidenciada como ações preventivas que contribuem para redução do óbito infantil por sufocação e engasgamento 22,23,28.

A maior parte dos óbitos investigados sofreu alteração da causa básica, corroborando com outro estudo realizado também no Recife/PE que avaliou a concordância da causa básica após a investigação dos óbitos infantis em geral ${ }^{29}$. Ainda é precária a qualidade do preenchimento da declaração de óbito no que se refere à falta de informações e distorções no preenchimento da causa básica. Autores afirmaram que a qualidade desse preenchimento é importante por permitir meIhor monitoramento e construção do perfil de mortalidade baseado na causa básica, contribuindo no planejamento de ações preventivas com objetivo de evitar o óbito infantil21,22,30.

De acordo com a lista de causas de morte evitável, os óbitos por causa externa deste estudo foram descritos em sua totalidade como evitáveis. A evitabilidade está relacionada às condições da assistência e do acesso oportuno aos serviços médicos. Para que seja possível alcançar maiores decréscimos no coeficiente de mortalidade infantil, torna-se fundamental identificar os principais

\section{REFERÊNCIAS}

1. Kyu HH, Pinho C, Wagner JA, Brown JC, Bertozzi-Villa A, Charlson FJ, et al. Global and national burden of diseases and injuries among children and adolescents between 1990 and 2013: findings from the global burden of disease 2013 study. JAMA Pediatrics, 2016; 170(3): 267-87.

2. Otterman G, Lahne K, Arkema EV, Lucas S, Janson S, Hellström-Westas L. Childhood death rates declined in Sweden from 2000 to 2014 but deaths from external causes were not always investigated. Acta paediatrica. 2019; 108(1):160-68.

3. Zonfrillo MR, Gittelman MA, Quinlan KP, Pomerantz WJ. aspectos de causas de morte e o potencial de redução e evitabilidade ${ }^{26-28 .}$

O estudo apresenta como limitação o pequeno número de causas específicas de morte, assim, não foi possível investigar tendências temporais. No entanto, os resultados encontrados são consistentes.

\section{CONSIDERAÇÕES FINAIS}

O presente estudo permitiu concluir que quanto menor o coeficiente de mortalidade infantil, maiores são as dificuldades encontradas para a continuidade de sua redução. Também foi observado que as mortes infantis por causas externas podem representar um empecilho na diminuição da mortalidade infantil em geral. Sabe-se que a redução do óbito por causa externa não é tarefa simples, por se tratar de um problema relacionado a múltiplos fatores que podem ser individuais, coletivos, estruturais ou organizacionais. Entretanto, a formulação e efetivação de políticas intersetoriais, sobretudo preventivas, que visem à redução desses óbitos, constituem um importante desafio. Por fim, a análise descritiva das características dos óbitos infantil por causa externa representa uma oportunidade para o desenvolvimento dessas políticas, planejadas segundo a multiplicidade de fatores que determinam o risco de morte por causa externa entre os menores de um ano.

Outcomes after injury prevention counselling in a paediatric office setting: a 25-year review. BMJ Paediatrics Open, 2018; 2(1):1-10.

4. Yamaoka Y, Morisaki N, Noguchi H, Takahashi H, Tamiya N. Comprehensive assessment of risk factors of cause-specific infant deaths in Japan. Journal of epidemiology, 2018; 28(6):307-314

5. Brasil MS. Internações Hospitalares do SUS-por local de internação-Ministério da Saúde-DATASUS. Acessado em janeiro de 2018. 
6. Gise R, Truong T, Parsikia A, Mbekeani JNA comparison of pediatric ocular injuries based on intention in patients admitted with trauma. BMC ophthalmology, 2019; 19(1):37.

7. Malta DC, Mascarenhas MDM, Silva MMAD, Carvalho MGOD, Barufaldi LA, Avanci JQ, Bernal, RTI. A ocorrência de causas externas na infância em serviços de urgência: aspectos epidemiológicos, Brasil, 2014. Ciência \& Saúde Coletiva, 2016; 2(1):3729-3744.

8. França EB, Lansky S, Rego MAS, Malta DC, França JS, Teixeira R, et al. Leading causes of child mortality in Brazil, in 1990 and 2015: estimates from the Global Burden of Disease study. Revista brasileira de Epidemiologia, 2017; 20(1):46-60

9. Malta DC, Sardinha L, Moura LD, Lansky S, Leal MDC, Szwarcwald CL, Duarte EC. Atualização da lista de causas de mortes evitáveis por intervenções do Sistema Único de Saúde do Brasil. Epidemiologia e Serviços de Saúde, 2010; 19(2):173-76.

10. Ramalho MODA, Frias PGD, Vanderlei LCDM, Macêdo VCD, Lira PICD. Avaliação da incompletude da declaracão de óbitos de menores de um ano em Pernambuco, Brasil, 1999-2011. Ciência \& Saúde Coletiva. 2015; 20:2891-98.

11. Sahu D, Nair S, Singh L, Gulati BK, \& Pandey, A. Levels, trends \& predictors of infant \& child mortality among Scheduled Tribes in rural India. The Indian J Medical Research, 2015; 141(5):709.

12. Squier W, Mack J, Jansen AC. Infants dying suddenly and unexpectedly share demographic features with infants who die with retinal and dural bleeding: a review of neural mechanisms. Developmental Medicine \& Child Neurology, 2016; 58(12):1223-1234

13. Caldas ADR, Santos RV, Borges GM, Valente JG, Portela MC, Marinho GL. Mortalidade infantil segundo cor ou raça com base no Censo Demográfico de 2010 e nos sistemas nacionais de informação em saúde no Brasil. Cad Saúde Pública, 2017; 33, e00046516.

14. De Sá GB. A cor da escravização ilegal de pessoas livres no Brasil oitocentista: Por uma tentativa de genealogia do artigo 179 do Código Criminal do Império. Revista brasileira de ciências criminais, 2017; (135):163-187.

15. Batista LE, Escuder MML, Pereira JCR. A cor da morte: causas de óbito segundo características de raça no Estado de São Paulo, 1999 a 2001. Rev Saúde Pública, 2004; 38:630-636.

16. Ahrens KA, Thoma ME, Rossen LM, Warner M, Simon AE. Plurality of birth and infant mortality due to external causes in the united states, 2000-2010. American journal of epidemiology, 2017; 185(5):335-344.

17. Fernandes LA, De Miranda DM, Ribeiro-Silva PC, Pereira DA, Salvador MG, Lage G M. Uma análise do desenvolvimento motor de crianças com transtorno do déficit de atenção e hiperatividade (TDAH). Revista Educação Especial, 2017; 30(57):115-127.

18. Durão C, Durão D, Pedrosa F. Mortes por Asfixias com Alimentos em Crianças: Aspectos Médico Legais da Sufocação Direta por Engasgamento. 2016.
19. Gao Y, Schwebel DC, Hu G. Measuring Trends in Infant Mortality Due to Unintentional Suffocation-Reply. JAMA Pediatrics, 2018; 172(9):887-888.

20. Abib SDCV, Françóia AM, Waksman R, Dolci MI, Guimarães HP, Moreira F,et al. Unintentional pediatric injuries in São Paulo. How often is it severe? Acta cirúrgica Brasileira, 2017; 32(7): 587-598.

21. Pimentel DEM, Fusco W. Caracterização do comportamento reprodutivo da população feminina de 20 a 49 anos da região nordeste em um contexto de baixa fecundidade-2000 a 2010. 2019; Anais, 1-12.

22. Andriano L, Monden $\mathrm{CW}$. The causal effect of maternal education on child mortality: Evidence from a quasi-experiment in Malawi and Uganda. Demography, 2019; 56(5): 1765-1790.

23. Son M, An SJ, Kim YJ. Trends of social Inequalities in the specific causes of Infant mortality in a nationwide birth cohort in Korea, 1995-2009. Journal of Korean medical science, 2017; 32(9):1401-1414.

24. Waqas M, Jooma R. Unintentional head injury in children less than 3 years of age: an insight into safety practices in a developing country. Pediatric neurosurgery, 2017; 52(5): 306-312.

25. Garcia LP, Fernandes CM, Traebert J. Risk factors for neonatal death in the capital city with the lowest infant mortality rate in Brazil. Jornal de pediatria, 2019; 95(2):194-200.

26. Heberlein EC, Picklesimer AH, Billings DL, Covington-Kolb $\mathrm{S}$, Farber N, Frongillo EA. Qualitative comparison of women's perspectives on the functions and benefits of group and individual prenatal care. Journal of midwifery \& women's health, 2016; 61(2):224-234

27. Fabiszewska AU, Zielińska KJ, Wróbel B. Trends in designing microbial silage quality by biotechnological methods using lactic acid bacteria inoculants: a minireview. World Journal of Microbiology and Biotechnology, 2019; 35(5):76.

28. Economou M, Kolokotroni O, Paphiti-Demetriou I, Kouta C, Lambrinou E, Hadjigeorgiou E, Middleton N. Prevalence of breast-feeding and exclusive breast-feeding at $48 \mathrm{~h}$ after birth and up to the sixth month in Cyprus: the BrEaST start in life project. Public health nutrition, 2019; 21(5):967-980.

29. Marques LJP, Pimentel DDR, Oliveira CMD, Vilela MBR, Frias PG, Bonfim CV D. Concordância da causa básica e da evitabilidade dos óbitos infantis antes e após a investigação no Recife, Pernambuco, 2014. Epidemiologia Serviços Saúde, 2018; 27, e20170557.

30. Ruoff $A B$, Andrade SRD, Piccoli T. O process de análise da evitabilidade dos casos de óbito infantil e fetal: estudo de caso único. Texto \& Contexto-Enfermagem, 2018; 27(4): e4030017.

\section{CORRESPONDÊNCIA}

Raphael Alves da Silva

Manoel Joaquim de Carvalho,

Alto da Estação, 22

Bonanç, Moreno- PE. CEP: 54800-000

E-mail: raphaelalves770@hotmail.com 